\title{
Leveraging MLC STT-RAM for Energy-efficient CNN Training
}

\author{
Hengyu Zhao \\ University of California, San Diego \\ La Jolla, California \\ h6zhao@eng.ucsd.edu
}

\begin{abstract}
Graphics Processing Units (GPUs) are extensively used in training of convolutional neural networks (CNNs) due to their promising compute capability. However, GPU memory capacity, bandwidth, and energy are becoming critical system bottlenecks with increasingly larger and deeper training models. This paper proposes an energy-efficient GPU memory management scheme by employing MLC STT-RAM as GPU memory to accommodate the image classification training workloads. We propose a data remapping scheme that exploits the asymmetry access latency and energy across soft and hard bits in MLC STT-RAM cells and the memory access characteristics in image classification training workloads. Furthermore, our design enables (i) energy-efficient memory access by leveraging bit-level similarity in training data and (ii) optimal feature map encoding to compress the contiguous 0s in feature maps. Our design reduces VGG-19 and AlexNet training time, GPU memory access energy and capacity utilization by $76 \%$ and $70 \%$, $45 \%$ and $40 \%, 26.9 \%$ and $26 \%$, respectively.
\end{abstract}

\section{INTRODUCTION}

Recent development of convolutional neural networks (CNNs) is radically altering the way we process various applications, such as image classification, speech recognition, object detection, and computer vision. Among these, image classification is one of the most widely targeted application domains in modern CNNs [32, 38, 39]. CNNs have two phases: training and inference - CNN models will be trained with input datasets during training phase and inference leverages the trained CNN models to infer things about new data it is presented with. As a result, training CNNs typically has much higher demand for compute power than inference. In fact, graphic processing units (GPUs) are extensively used to accelerate the training of modern $\mathrm{CNN}$ models, due to their promising compute horsepower and strong backend support for training software libraries and frameworks $[1,3]$.

Software developers strive to improve CNN training performance and accuracy by designing efficient $\mathrm{CNN}$ algorithms and training frameworks $[8,28,41]$. This is often achieved by adopting larger and deeper CNNs with more parameters. However, such scaling of the training networks makes training workloads increasingly data intensive. GPU memory capacity, bandwidth, and access energy

Permission to make digital or hard copies of all or part of this work for personal or classroom use is granted without fee provided that copies are not made or distributed for profit or commercial advantage and that copies bear this notice and the full citation on the first page. Copyrights for components of this work owned by others than the author(s) must be honored. Abstracting with credit is permitted. To copy otherwise, or republish, to post on servers or to redistribute to lists, requires prior specific permission and/or a fee. Request permissions from permissions@acm.org.

MEMSYS, October 1-4, 2018, Old Town Alexandria, VA, USA

(c) 2018 Copyright held by the owner/author(s). Publication rights licensed to ACM. ACM ISBN 978-1-4503-6475-1/18/10..\$15.00 https://doi.org/10.1145/3240302.3240422.

\author{
Jishen Zhao \\ University of California, San Diego \\ La Jolla, California \\ jzhao@eng.ucsd.edu
}

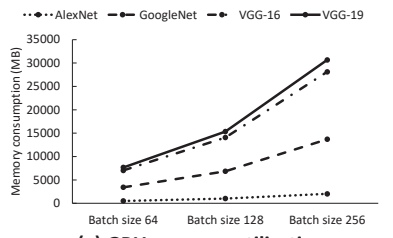

(a) GPU memory utilization.

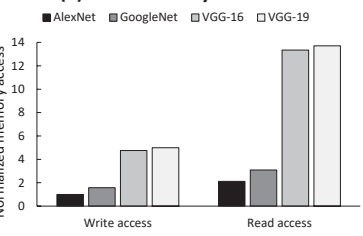

(c) GPU memory access.

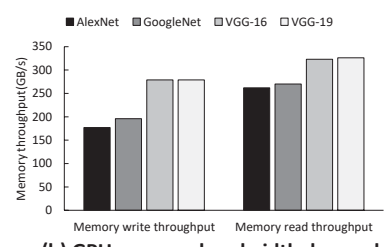

(b) GPU memory bandwidth demand

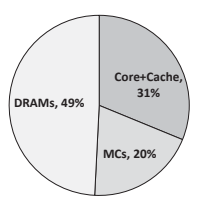

(d) NVIDIA GTX 1080 Ti dynamic power breakdown when executing VGG-19.
Figure 1: GPU memory demand and system power breakdown.

are becoming critical system bottlenecks in $\mathrm{CNN}$ training [17, 30, $36,40]$.

Figure 1(a) shows the memory capacity demand of CNNs that are recent winners of the ImageNet Large Scale Visual Recognition Challenge(ILSVRC) [7], including AlexNet [32], GoogLeNet [39], VGG-16 and VGG-19 [38] (experimental setup is described in Section 5). AlexNet [32] only contains 8 layers in total, with five convolutional layers and three fully connected layers. Therefore, it has low memory capacity utilization. However, one of the most recently developed image classification CNNs, VGG-19 [38], employs 16 convolutional layers and three fully connected layers. As a result, VGG-19 has high memory capacity demands. When increase the batch size to 128, even a single NVIDIA's GTX 1080Ti (11 GB device memory) [6] cannot meet the memory demand. The memory demand can further increase with the design of recent ILSVRC winner, which adopts more than a hundred convolutional layers [25].

Moreover, the increase of memory capacity demand also increases the bandwidth demand and dynamic energy consumption in GPU device memory. GPU processors typically have small-capacity storage resources, such as caches, register files, and shared memories. The huge working set of CNN training workloads will not fit in GPU processors. As a result, the capacity demand also leads to high memory bandwidth demand as demonstrated in Figure 1(b). In addition, the training workloads are more read intensive than write intensive (Figure 1(c)). Figure 1(d) shows a power breakdown of various components in NVIDIA's GTX 1080Ti GPU system, when executing VGG-19 (Section 5 describes our power and energy modeling). It demonstrates that GPU device memory can consume a critical portion of the total power in GPU systems. In order to maintain reasonable training performance with the continuous scaling 


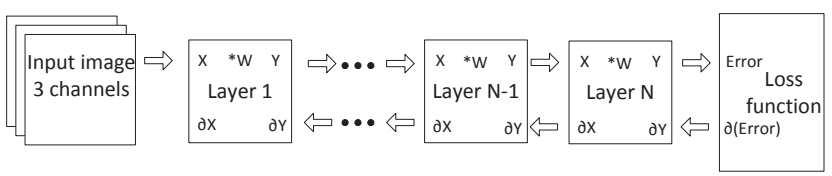

Figure 2: CNN architecture.

of CNN models, it is critical to ensure that future GPU systems can keep up with the increase of the memory demand.

Most previous works focus on optimizing software-based mechanisms to tackle the memory bottlenecks. Whereas most previous studies demonstrate that increasing the compute batch size and the number of network layers can improve the accuracy of training, the GPU memory constraint can force us to approach the other way. In order to work around the GPU memory bottlenecks, several recent studies suggest to use less desirable network architectures (e.g., with smaller number of layers and batch sizes), adopt memory-efficient but lower-performance convolutional algorithms, or distribute the CNN across multiple GPUs [17, 31, 40]. A recent study designs a runtime memory manager that allows GPU to borrow CPU memory to store training data [36]. Doing so can significantly increase the effective memory capacity for GPU systems, yet at the cost of reducing performance and energy-efficiency of training.

The goal of this paper is to improve the GPU performance, energy efficiency, and capacity utilization of $\mathrm{CNN}$ training for image classification, without sacrificing on any of these metrics. Also, this paper will not change the model accuracy, because we do not change the values of training data. To this end, we design a multi-level cell (MLC) STT-RAM-based GPU device memory with lightweight modifications to the memory controllers and memory banks. We propose a data remapping scheme, which reduces memory traffic and access latency by categorizing and mapping different types of data in different manners in MLC STT-RAM. Our scheme further enables two GPU memory optimization mechanisms: i) an energyefficient memory access mechanism, which reduces memory access energy consumption by avoiding the unnecessary writes at the bit level; ii) a sparsity-aware data encoding mechanism, which increases effective memory capacity with data encoding by exploiting the sparsity in feature maps. This paper makes the following contributions:

- We propose a data remapping scheme that stores various types of data of training workloads in MLC STT-RAM, based on their different access characteristics. Our remapping scheme enables energy and capacity efficient MLC STT-RAM data access.

- We present two memory access optimization mechanisms based on our data remapping scheme: i) BitLevel-leverage the asymmetric write current and access latency; ii) SparseCode-exploits the sparsity in feature maps to compress the data without quality loss.

- We develop a set of lightweight hardware implementations and software support to facilitate our mechanisms.

\section{BACKGROUND AND MOTIVATION}

This section provides background on CNN architectures and MLC STT-RAM. We also motivate our work by discussing our observations on CNN image classification training workloads and MLC STT-RAM technology.

\subsection{Convolutional Neural Networks}

Deep neural networks (DNNs) have many types, such as recurrent neural networks (RNNs), convolutional neural networks (CNNs), etc. This paper focuses on the feedforward style CNNs commonly found in AlexNet [32], GoogLeNet [39], and VGG [38], which is mainly used in computer vision, and image recognition, image classification.

CNNs consist several layers, including convolutional layers that perform image convolution, activation layers to make neural networks nonlinear with activation functions, pooling layers to reduce the feature map size by down sampling, and fully connected layers to analyze features and classify input images into groups. CNNs have two phases: training and inference. Training allows CNNs to learn and update weights with multiple layers of neural networks, through forward and backward propagations with opposite traverse directions (Figure 2). Forward propagation generates feature maps using weights and backward propagation updates the weights. Inference employs the trained models to perform new recognitions or classifications. This paper focuses on studying the training phase, which is typically much more compute and data intensive than inference phase.

Forward propagation. Forward propagation is performed from the first layer to the last layer. As shown in Figure 2, each layer multiplies its input feature map $x$ by multiple convolutional filters and outputs $y$ to the next layer as its input feature map. An activation layer or a pooling layer are also applied to the following results to realize their functions as mentioned above, and this process is repeated until the training is over.

Backward propagation. As shown in Figure 2, when forward propagation of the last layer completes, the loss function generates output that is calculated by feature maps of the previous layer and validation dataset. Then, the gradient map is obtained by chain rule:

$$
\frac{\partial \text { Error }}{\partial Y_{(N-1)}}=\frac{\partial \text { Error }}{\partial Y_{(N)}} \cdot \frac{\partial Y_{(N)}}{\partial Y_{(N-1)}}
$$

Where Error is the sum of the network's prediction error over all training examples, $Y_{(N-1)}$ is the output feature map of layer $(N-1)$, and $Y_{(N)}$ is the output feature map of layer $N$.

Because the output $\frac{\partial E r r o r}{\partial Y_{(N-1)}}$ is the product of the input $\frac{\partial E r r o r}{\partial Y_{(N)}}$ and $\frac{\partial Y_{(N)}}{\partial Y_{(N-1)}}$, this derivation step can require reading feature maps from memory and writing updated weights into the memory. With the chain rule, layer $(N-1)$ can then get its own gradient map $\partial Y_{(N-1)}$ with its feature map $Y_{(N-1)}$, and pass the $\partial Y_{(N-1)}$ to layer $(N-2)$ as its input. When backward propagation reaches to the first layer, weights of all layers need to be updated with new values for this whole iteration.

In feed forward style neural network, backward propagation is also performed sequentially similar to forward propagation. A typical iteration includes both forward propagation to generate feature maps and errors, and backward propagation to calculate gradient maps and update weights. Thus, training a neural network is composed of millions of repeated iterations, until the gradient map approaches to zero, which is known as gradient descent [10]. Based on the characteristics of backward propagation algorithms, 


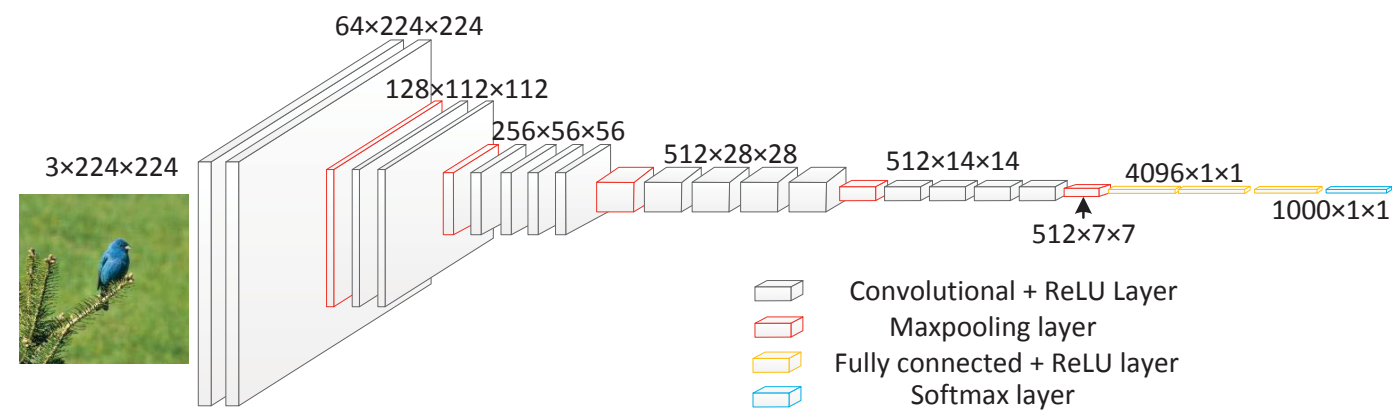

Figure 3: VGG-19 architecture.

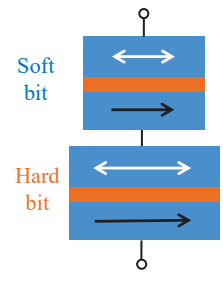

(a)

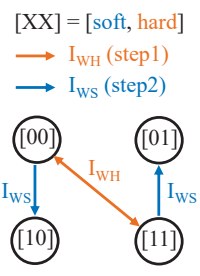

(b)

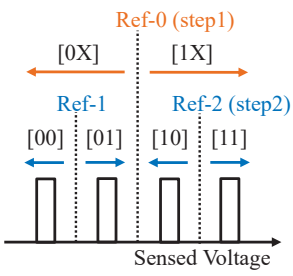

(c)
Figure 4: Multi-level cell STT-RAM. (a) Series MLC MTJ structure; (b) Two-step write operation; (c) Two-step read operation.

training the total training set at one time will be much more helpful to efficiently obtain the optimal solution. However, due to the large sizes of training sets in modern CNNs, we have to train CNNs in batching style. In order to obtain better training performance, larger batch size is highly recommended, thus resulting in higher memory capacity and bandwidth demand than smaller batch sizes.

Input images and feature maps. Input images of image classification training workloads can be color digital images, which typically consist of multiple matrixes of pixels consisting of three different color channels: red (R), green $(G)$, and blue (B). Each channel can be represented by a 8-bit binary number, i.e., each pixel is stored as a three-byte value in the memory. Feature maps are the results after several computations of input images; they consists of data blobs that contain multi-dimensional feature information, but stored in the memory as 2D arrays. Figure 3 shows the computation flow of VGG-19 and the process of input images and feature maps. Each input image will generate multiple feature maps after convolution computation. The number of feature maps equals the number of convolution kernels of each layer. For instance, CONV1 layer can generate 64 feature maps with its convolution kernels. At the beginning of the training, input images can be resized to a desired uniformed size. For example, VGG-19 requires all input images to be converted to $224 \times 224$ (Figure 3).

\subsection{MLC STT-RAM}

Multi-level cell (MLC) STT-RAM cells store multiple logic bits (typically two bits) in each cell, increasing the density of STT-RAM (Figure 4). Also, MLC STT-RAM has great endurance. There are two categories of MLC MTJ designs, series [26] and parallel [19]. Series MLC STT-RAM has two MTJs stacked together to achieve four resistance states by the combinations of their magnetization directions, as shown in Figure 4(a). In parallel MLC MTJs, the free layer has two domains to represent two logic bits. Our design leverages the series MLC STT-RAM. Series MLC STT-RAM has been demonstrated to be more feasible than the parallel implementation, because series design is compatible with advanced MTJ technologies, such as perpendicular MTJ and has overwhelming advantages in read and write reliability. Instead, the parallel implementation is only applicable to in-plane MTJ technology [20].

Read and write operation of MLC STT-RAM cell. In Figure 4(a), the two MTJs of series MLC MTJ structure have different areas to distinguish the two logic bits. The bit stored in the smaller MTJ is a soft bit; the bit in the bigger MTJ is a hard bit. Given a constant resistance-area product and a critical switching current density $\left(I_{C}\right)$, the soft bit has a higher resistance than the hard bit. As such the soft bit is typically the more significant bit (MSB), and it requires a smaller switching current, i.e. $I_{C \text {, soft }}<I_{C}$, hard . The write and read operations of series MLC STT-RAM are illustrated in Figure 4(b) and (c), both of which contain two steps. As an example, in a write operation, in the first step a large current $I_{W H}\left(I_{W H}>I_{C \text {, hard }}\right)$ is applied to switch the hard bit; at the same time the soft bit gets the same value as the hard bit; in the second step a smaller current $I_{W S}\left(I_{C \text {, soft }}<I_{W S}<I_{C \text {, hard }}\right)$ is used to flip only the soft-bit. Figure 4(c) illustrates a two-step read operation based on voltage sensing, which requires three reference voltages (Ref- 0 , Ref- 1 , and Ref-2) and two comparisons. In the first step, the soft bit is detected by comparing the sensing voltage with Ref- 0 . In the second step, the hard bit is read by comparing the sensing voltage with either Ref- 1 or Ref- 2 based on the result of the first step.

MLC STT-RAM as main memory. STT-RAM has been considered as a promising replacement of DRAM in main memory system design. Compared with DRAM, STT-RAM has many advantages, such as lower leakage power and no radiation-induced soft errors. With the MLC technology, the density can be doubled compared with SLC STT-RAM. MLC PCRAM also offers high density and low leakage benefits. However, the technology impose much longer access latency and higher dynamic energy than DRAM and MLC STT-RAM. Furthermore, the writing mechanisms of MLC PCRAM cells do not have the unique properties as MLC STT-RAM has. As such, we adopt MLC STT-RAM as our main memory technology in our design. 


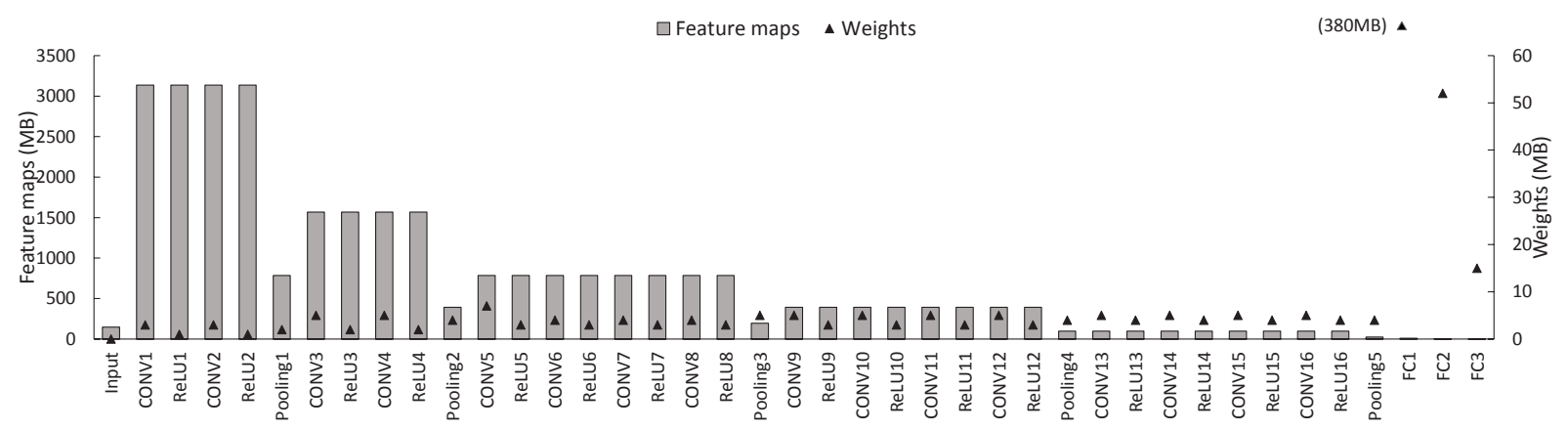

Figure 5: VGG-19 memory utilization in each layer.

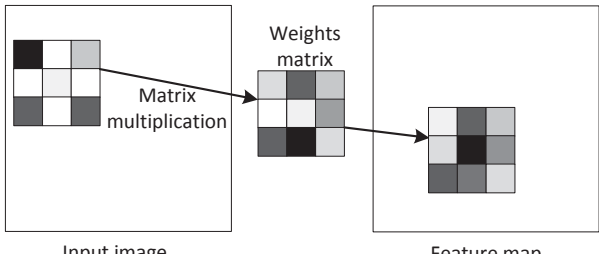

(a) 2D convolution

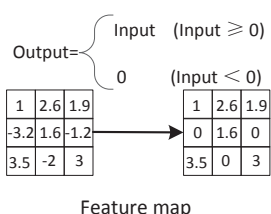

(b) ReLU function

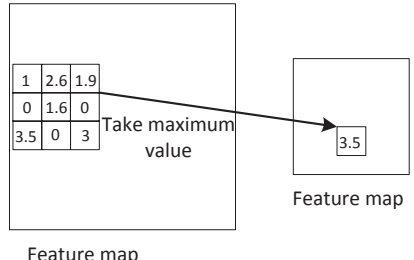

(c) Maxpooling

Figure 6: Basic functions of CNNs.

\subsection{Motivation}

We motivate our proposed design mechanisms by the following observations:

Feature maps cost most of memory capacity. The total volumes of input images can be huge. However most of the input images are stored in the disk or SSD, when not being accessed; only a single batch of input images is fetched into memory at a time. Rather, we observe that the majority memory space is used to store feature maps across convolutional layers as shown in Figure 5. Feature maps have much lower memory utilization in fully connected layers. In these layers, weights are the primary memory capacity consumers. However, the total memory capacity utilization of fully connected layers appear much lower than convolutional layers. Therefore, improving the capacity utilization and energy efficiency of feature map access and storage can significantly improve GPU memory system performance and energy efficiency.

Impact of CNN functions on neighbor data similarity. Figure 6 shows three basic CNN functions. First, A 2D convolution will generate feature maps through matrix convolution with convolution filters. Second, the following ReLU function can increase the similarity of feature maps. An intermediate feature map can have both negative and positive data in it, and ReLU functions in image classification applications, such as VGG-19, typically only change negative values to zeros. As a result, the newly generated feature maps can have a large portion of 0 s gathered together after applying a ReLU function. This will increase the neighbor data block similarity in feature maps. Finally, a maxpooling function takes the maximum value in each window of certain sizes and generate a new feature map with a smaller size. As a result, data with large values can be clustered together. It is likely that the higher order bits share the same bit values. As such, the feature maps generated across the $\mathrm{CNN}$ functions offer us with substantial opportunity of exploiting both bit-level and data block level similarity.

CNNs are read intensive applications (Figure 1(c)). In CNN training, the number of memory read accesses is much higher than the writes. As MLC STT-RAM is reported as having promising single-step read performance and energy consumption $[9,11,13$, 16], using this emerging memory technology offers opportunity of improving the overall performance of CNN training.

Issues and opportunities with MLC STT-RAM cell. Due to the two-step read and write operations, MLC STT-RAM has longer access latency and higher access energy than SLC STT-RAM. This can degrade system performance and energy efficiency, despite MLC STT-RAM's density benefits. However, we observe that, reading the soft bit only takes one step, while writing the soft bit only requires a small switching current that will not flip the hard-bit. Thus, MLC STT-RAM can perform in a similar way as SLC by only accessing the soft-bits, which improves both access speed and the energy efficiency. Furthermore, MLC STT-RAM has a write disturbance issue: writing the hard bit with a large write current can also change the soft bit in the memory cell to be the same value. However, these results in the opportunity of value depended write current in MLC STT-RAM cells: if both bits in a cell need to be changed and the new value is either " 00 " or " 11 ", only a one-step write with large write current is necessary. Otherwise, if the new values of the soft bit and the hard bit are different from each other (i.e., writing "01" or "10"), a two-step write with longer latency is required. Such opportunities motivate us to explore the use of MLC STT-RAM in feature map storing and remap the data in the memory to exploit the value depended write property. 
Table 1: Overview of categories of data in MLC STT-RAM, remapping schemes, and access mechanisms.

\begin{tabular}{|c|c|c|c|c|}
\hline Data Type & $\begin{array}{c}\text { Memory } \\
\text { Utilization }\end{array}$ & Reuse & Remapping Scheme & Access Modes \\
\hline Dense feature map (CONV layers) & High & Write once, multiple reads & Remap across soft and hard bits & BitLevel \\
\hline Sparse feature map (CONV layers) & High & Write once, multiple reads & Remap across soft and hard bits & SparseCode + BitLevel \\
\hline Feature map (FC layers) & Low & Write once, multiple reads & Remap to soft bits & Fast soft bit access \\
\hline Weights (CONV layers) & Low & Multiple reads and writes & Remap to soft bits & Fast soft bit access \\
\hline Weights (FC layers) & High & Multiple reads and writes & Remap across soft and hard bits & BitLevel \\
\hline Input images and gradient maps & Low & Write once, read once & Remap to soft bits & Fast soft bit access \\
\hline Other & Low & Multiple reads and writes & No remapping & Normal access \\
\hline
\end{tabular}

\section{OUR DESIGN}

The goal of our design is to reduce the GPU memory capacity utilization and energy consumption, while improving the performance of $\mathrm{CNN}$ training. To this end, we propose an data remapping scheme that efficiently maps various types of training data across GPU main memory regions and the bits in each memory cell, based on characterization of various types of training data stored in the memory. Furthermore, our design also enables two optimization mechanisms, BitLevel and SparseCode, to improve the performance and energy efficiency of training data access. Hardware implementation and software interface of our design will be discussed in Section 4 .

\subsection{Data Remapping and Access Modes}

Table 1 lists major categories of data stored in GPU memory based on our workload profiling. Feature maps and weights are two critical categories of data stored in GPU memory (Figure 5). In particular, feature maps are the primary memory consumer in convolutional layers, while weights utilize the most of the GPU memory space in fully connected layers. Note that high memory capacity utilization typically leads to high GPU memory bandwidth utilization [36]. Table 1 also illustrates our proposed data remapping scheme and optimized data access modes of each type of data.

Feature map. As feature maps are the results after several computations of the same set of input images. We observe that neighbor data blobs (groups of 32-bit floating point values) in feature maps can have similar or even the same bit values, because the features are extract from the input digital images. Based on this observation, we provide the following options for remapping and accessing feature maps:

- Option 1: Dense feature maps (without many contiguous 0s) in convolutional layers (including ReLU and pooling layers) Remap the feature map data in a manner, where every two corresponding bits in a pair of neighbor data blobs are stored in the soft and hard bits in each memory cell; As a result, the soft and hard bits of the same memory cell can have the same bit value, which enables our BitLevel optimization mechanism (details discussed in Section 3.2)

- Option 2: Sparse feature maps (with a large number of contiguous 0s) in convolutional layers - Encode the feature maps with SparseCode (Section 3.3), and then remap the feature map data the same manner as Option 1. Sparse feature maps are commonly found in the workloads that we evaluate.
- Option 3: Feature maps in fully connected layers - Write them into soft bits to reduce the access energy and latency, as feature maps have insignificant memory utilization in fully connected layers.

In convolutional layers, we employ the Option 1 by default. In case the memory controller identifies substantially low number of contiguous $0 \mathrm{~s}$ in the first 65536 data blocks in feature map writing flow (details discussed in Section 4), our design will switch Option 2. The switch of remapping schemes between convolutional and fully connected layers does not require data migration - we simply write the output feature maps of a convolutional computation to the soft bits.

Weights. Weights have low memory utilization in convolutional layers. Therefore, we remap them in the soft bits to reduce access latency and energy. In fully connected layers, where weights are the primary memory space consumer, we observe that weights also have substantial neighbor bit-level value similarity. Therefore, we remap the weights to soft and hard bits of MLC STT-RAM cells in a similar way as feature maps in convolutional layers. The difference is that neighbor weights are simply a pair of 32-bit floating point values, instead of larger-granularity data blobs.

Input images and gradient maps. Input images and gradient maps have much lower memory utilization than feature maps [36]. In particular, input images and gradient maps consume up to $6 \%$ and $7 \%$ the memory space used to store feature maps, respectively, in our workloads. Furthermore, input images are only accessed at the beginning of the workload; the corresponding memory space is recycled once the images are read. In backward propagation, the generated gradient map for layer $\mathrm{N}+1$ is no longer useful once the backward propagation reaches layer N. GPU systems can potentially recycle the corresponding memory space. Therefore, we remap such data in soft bits of memory cells to ensure low access latency and energy.

Other data. CNN workloads can also generate other types of data, such as parameters and intermediate results of compute kernels. However, these data consumes insignificant space and access energy in GPU memory compared to the above types of data [36]. Therefore, we store such data in normal manner without data remapping.

\subsection{BitLevel: Energy-efficient Access with Bit-level Storage}

We observe that naively replacing GPU device memory with MLC STT-RAM (as shown in Figure 7(a)) will require two-step writes 

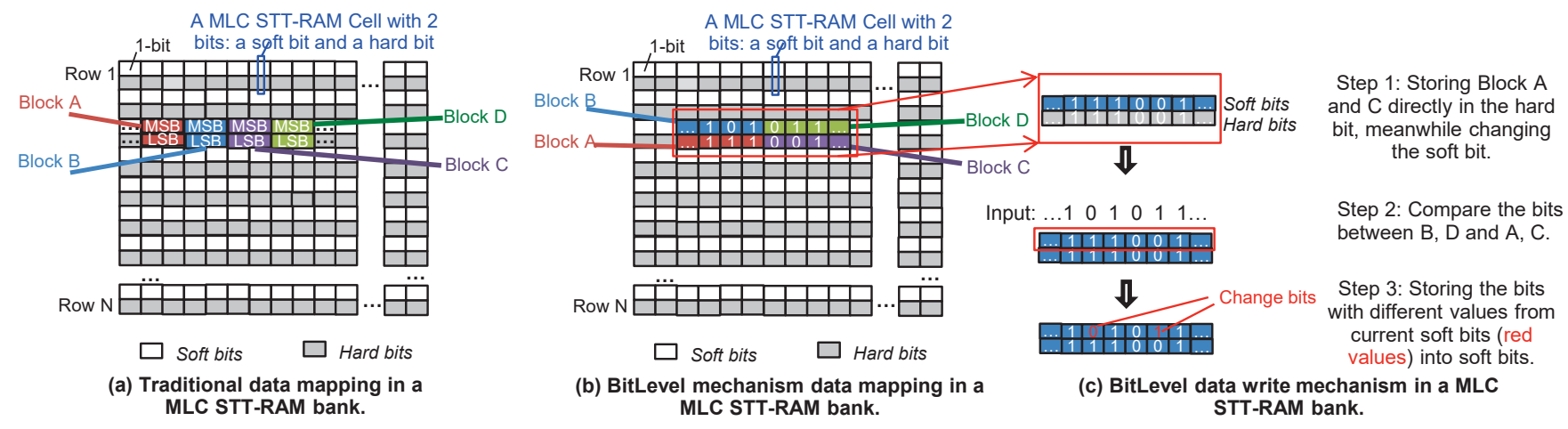

Figure 7: Energy-efficient access with bit-level storage (BitLevel mechanism).

and reads of most memory cells due to the different values to be written into each cell (Section 6). In order to reduce the energy consumption and bandwidth demand of MLC STT-RAM access, we propose an energy-efficient MLC STT-RAM access mechanism with bit-level data storage. This optimization mechanism leverages i) the write disturbance in MLC cells, ii) the asymmetric access energy across soft and hard bits, and iii) the bit-level value similarity in feature maps and weights.

Figure 7 illustrates an example employing this mechanism. Assuming four contiguous data blocks A, B, C, and D (e.g., data blobs in feature maps or 32-bit floating point values in weights), traditional memory storage mechanism will directly map the data based on its physical address with the MSBs in soft bits and LSBs in hard bits (Figure 7(a)). It is likely that the two bits written into the same cell belong to random locations in the feature map or weight matrices. Therefore, a large portion of memory cells require two steps to write.

Instead, this BitLevel mechanism remaps the data blocks as shown in Figure 7(b). We store neighbor data blocks (e.g., A and B) in the soft and hard bits of the same memory cells. Based on our profiling of feature maps (Section 6), values of neighbor data blocks can appear similar with significant amount of same bit values. This is because the neighbor data blobs in a feature map tends to have similar feature characteristics (Section 2.3). Due to write disturbance in MLC STT-RAM, a single write to the hard bit (e.g., a bit in Block A) will also write the same value to the soft bit (i.e., corresponding bit in Block B) in the same cell. Therefore, a single step write to the hard bits will write all the bits of Block A, along with substantial amount of bits in Block B. We only need to perform a second-step write to flip several soft bits with different values from the corresponding hard bits in the same cell. Figure 7(c) illustrates the steps required to write to our remapped memory space. Furthermore, we also observe high bit-level value similarity with our data remapping mechanism used in fully connected layers (Section 6), where weights have the most significant memory utilization. As such, our mechanism also improves the energy efficiency of weight access in these layers.

The BitLevel mechanism significantly reduces the latency and energy consumption of GPU memory access. The performance and energy consumption improvement in memory access also improves the energy efficiency of workload execution on GPU systems. Section 6 quantitatively demonstrates the performance and energy benefits of our design.

\subsection{SparseCode: Sparsity-aware Feature Map Encoding}

To further improve the memory capacity usage and access energy efficiency, we develop a sparsity-aware feature map encoding mechanism that compresses the feature maps stored in memory. This mechanism exploits the sparsity in feature maps yielded by ReLU functions to encode the large amount of $0 \mathrm{~s}$.

With workload characterization, we identify that the number of $0 \mathrm{~s}$ in feature maps is significantly increased after applying ReLU functions. Furthermore, the 0 s tend to gather in contiguous memory areas as shown in Figure 8(a) (if the feature maps are visualized as images, these will appear to be areas in black color). As such, we employ a sparsity-aware feature map encoding mechanism to compress the feature maps.

Encoding mechanism. To this end, we adopt two data structures, index and data (similar to a previous study [34]), to store feature maps in the memory. The data stores each non-zero values, while the index records the number of contiguous 0s between two nonzero values. Our evaluation (Section 6) adopts 4-bit integer indexes, because our workloads have up to 15 contiguous 0 s between two non-zero values in common case. Data are 32-bit floating point values. As shown in Figure 8(b), each data (black number) is followed by an index (red number) in the encoded format. Such encoding mechanism does not require extra metadata to identify across index and data when reading the feature maps, because the every 32-bit data is uniformly followed by a 4-bit index. Note that weights can be compressed with the same encoding policy. However, we do not observe substantial energy and performance improvement by compressing weights, due to their low memory utilization in convolutional layers. Therefore, our design only employ the SparseCode mechanism on feature maps.

Mapping indexes and data to leverage BitLevel mechanism. After encoding the feature maps, we store the encoded data and index across the soft and hard bits using the BitLevel mechanism 3.2. As shown in Figure 8(c), in order to increase the bit-level similarity in soft and hard bits, we store neighbor indexes in soft and hard bits 


\begin{tabular}{|l|l|l|l|l|l|}
\hline 1 & 0 & 2 & 0 & 9 & 9 \\
\hline 0 & 0 & 0 & 5 & 0 & 1 \\
\hline 0 & 0 & 0 & 8 & 7 & 8 \\
\hline 0 & 6 & 0 & 0 & 2 & 0 \\
\hline 0 & 0 & 2 & 0 & 0 & 0 \\
\hline 7 & 0 & 3 & 3 & 0 & 0 \\
\hline
\end{tabular}

(a) An example of a $6 \times 6$ feature map.
102099; 000501; 000878; 060020; 002000; 703300

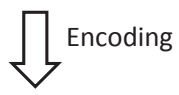

112190935113807081622323713032

Black digits are data. Red digits are indexes, and each index takes four bits.

(b) SparseCode mechanism.

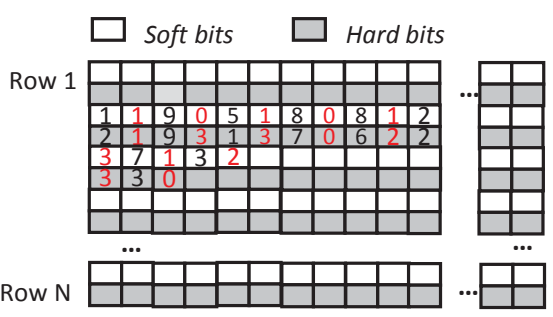

(c) Mapping encoded index and data in a MLC STT-RAM bank.

Figure 8: Sparsity-aware feature map encoding (SparseCode) mechanism.

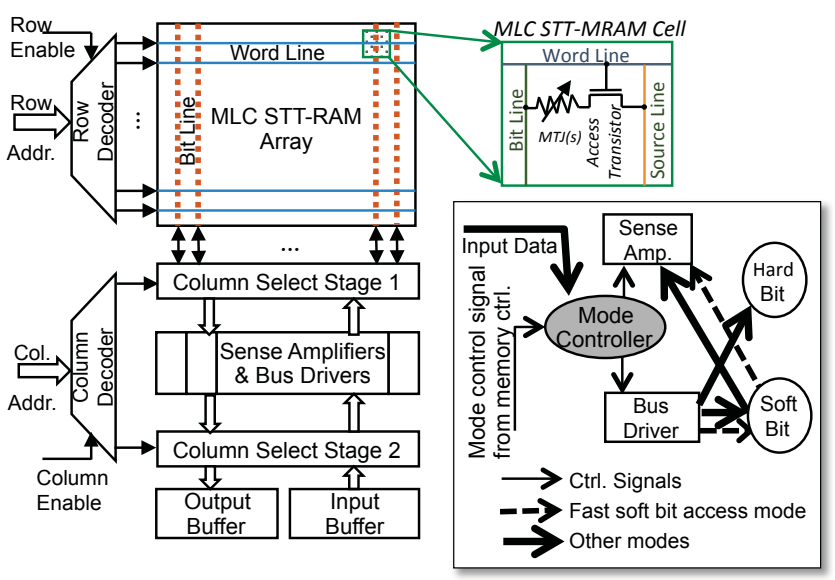

Figure 9: MLC STT-RAM bank organization.

of the one set of cells, while storing neighbor data blocks in another set. In fact, the encoded data can have substantial neighbor block value similarity. For example, the indexes are 4-bit integers. The neighbor indexes are highly possible to share substantial amount of the same bit-level values.

\section{IMPLEMENTATION}

In this section, we discuss hardware implementation and software support required by our design.

\subsection{Memory Bank Organization}

Figure 9 illustrates an MLC STT-RAM bank incorporated with the components that implement our design mechanisms. We implement two modifications to the memory banks. First, we add a mode controller implemented by a set of switches to distinguish the fast soft bit access mode with other modes that access both soft and hard bits. Second, we adopt an optimized MLC STT-RAM peripheral circuit design [13] to implement the three-step energy-efficient writes in our BitLevel mechanism (Figure 7(c)). By adjusting the biases on the word line and bit line, the circuit design allows a writing current with different amplitudes and directions to be applied to an MTJ. It is also possible to avoid writing a memory cell, if the original soft and hard bit values of the cell are the same as the new values. However, doing so can introduce extra read operations to

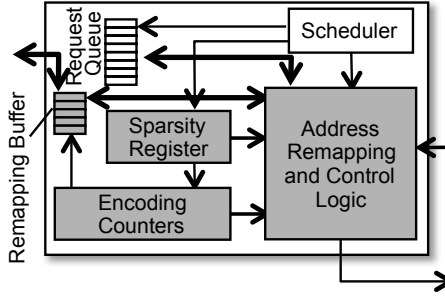

Software hints:

00: Feature maps in CONV layers

01: Weights in FC layers

10: Feature maps in FC layers, weights in CONV layers, input images, or gradient maps 11: Other data

Mode ctrl. Signals: $0:$ Fast soft bit access 1: Other access modes

Figure 10: Memory controller design. Modifications are represented by shaded components.

compare the original values with the new values. Therefore, our design does not consider such optimizations. The rest of the memory bank organization stays the same as conventional MLC STT-RAM designs.

\subsection{Memory Controller Design}

Our design requires the memory controller support to 1) perform physical address remapping, 2) generate control signals of various access modes, 3) choose between Option 1 and Option 2 of feature map access, and 4) encode/decode the data flow of feature maps accessed by the SparseCode mechanism. Figure 10 illustrates our modification to memory controllers.

Address remapping and control logic. We add an address remapping logic in the memory controller to calculate the new physical address of data accesses, based on our address remapping scheme and software hints of data categories (software support is discussed in Section 4.3). The address remapping logic also generates access mode control signals, which distinguish between access to the soft bits only and those to both bits in memory cells. In addition, we implement encoding and decoding logic used by our SparseCode mechanism. The encoding logic inserts the indexes generated by encoding counters among non-zero data values; the decoding logic inserts $0 \mathrm{~s}$ into the request queue according to the indexes. Figure 11 shows the control flow of the address remapping and control logic.

Remapping buffer. We adopt a buffer to store the remapped memory requests (output to or input from the MLC STT-RAM). The size of each buffer entry is the same as the request queue entry. The buffer also stores encoded feature map data, when accessed by SparseCode mechanism. We implement a 16-entry buffer, which is sufficient based on our performance evaluation. 


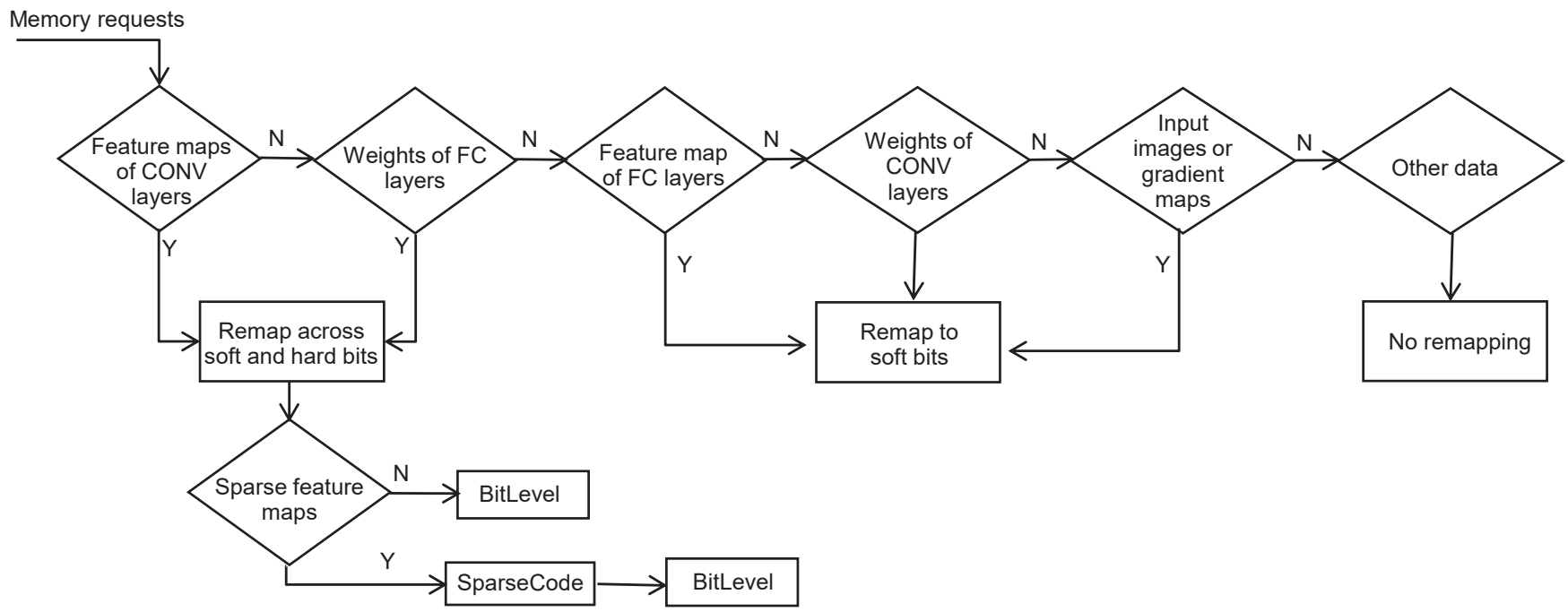

Figure 11: Control flow of logic design.

Sparsity register. We employ a 16-bit register to determine whether a feature map is sufficiently sparse for adopting the SparseCode mechanism. The register counts the number of non-zero values with an index that is smaller than a predefined threshold. After investigating the first $65536\left(2^{16}\right)$ accessed data values, we identify that a feature map is dense if the register value is larger than certain threshold. In our evaluation, most of the feature maps are identified as sparse when we set the thresholds of index and register values to be eight and 32768 , respectively.

Encoding counters. We add a 4-bit counter to count the number of $0 \mathrm{~s}$ between two non-zero values in the data flow sent to each memory bank. Our SparseCode mechanism employs the counter results to generate index values. Whenever reaching a non-zero value in the data flow, we simply reset the counter. If there are more than 15 continuous 0 s, the counter not only should be reset but also change the following data value to 0 .

\subsection{Software Support}

Our design requires software hints on the categories of data being accessed. This can be implemented as annotation mechanisms implemented in CNN frameworks, such as Caffe [28]. For example, before a convolutional layer outputs a feature map, the software can provide a pointer to the first data blob and the size of the feature map. Based on such information, the address remapping logic in the memory controller can calculate the address range of the feature map.

\section{EXPERIMENTAL SETUP}

Our evaluation combines real-machine profiling with performance and energy modeling.

\subsection{Workloads}

VGG-19 and AlexNet. Our experiments evaluate VGG-19 [38] and AlexNet [32], two of the latest winners of ILSVRC [7]. The applications perform image classification and localization. The workloads are widely used in recent studies on CNN training and image classification [30,36].

Datasets. We employ ImageNet as our training datasets. It is a huge image dataset which contains millions of images belong to thousands of categories. Beginning at 2010, ILSVRC [7] has been held annually. ILSVRC exploits a subset of ImageNet with 1.3 million training images, 50000 validation images, 150000 testing images in 1000 categories. Every category has about 1300 of training images and 50 validation images.

Training framework. We adopt Caffe [28] as our training framework. Caffe is a widely-used deep learning framework developed by Berkeley AI Research.

\subsection{Real Machine Configuration}

We run VGG-19 and AlexNet on NVIDIA GeForce GTX 1080Ti graphic card [5] with Dell Precision T7810 Tower Workstation. GTX 1080Ti graphic card has 3584 CUDA cores, 11GB DRAM device memory, $484 \mathrm{~GB} / \mathrm{s}$ memory bandwidth, and Pascal architecture. Table 2 lists the details of our system configuration. We use NVIDIA's profiling tools [2] to collect performance and power statistics.

\subsection{Performance and Energy Modeling}

Our evaluation adopts the MLC STT-RAM cell performance, energy, and area parameters provided by prior MLC STT-RAM designs [ 9 , $11,16]$. We scale the parameters to $32 \mathrm{~nm}$ technology node the same way as previous studies [13]. We employ NVSim [18] to calculate the required MLC STT-RAM parameters as listed in Table 3. We tune the device and circuit models in NVSim [18] to reflect the characteristics of MLC STT-RAM designs. DRAM energy and area parameters are modeled based on the Micron GDDR5X specifications [4]. We 
Table 2: System configuration.

\begin{tabular}{|c|c|}
\hline CPU & Intel Xeon E5-2620 V3@2.4GHz \\
\hline Main memory & 16GB DDR4 \\
\hline Operating system & Ubuntu 16.04.2 \\
\hline \hline GPU & NVIDIA GeForce GTX 1080 Ti (Pascal) \\
\hline GPU cores & 28 SMs, 128 CUDA cores per SM, 1.5GHz \\
\hline L1 cache & 24KB per SM \\
\hline L2 cache & $4096 \mathrm{~KB}$ \\
\hline Memory interface & 8 memory controllers, 352-bit bus width \\
\hline GPU main memory & 11GB GDDR5X \\
\hline
\end{tabular}

Table 3: Memory access parameters comparison.

\begin{tabular}{|c|c|c|}
\hline & MLC STT-RAM & DRAM \\
\hline Read energy (pJ/bit) & 0.51 & 1.39 \\
\hline Write energy (pJ/bit) & $\begin{array}{c}\text { Soft Transition: 1.81 } \\
\text { Hard Transition: 2.69 }\end{array}$ & 0.33 \\
\hline Read speed (ns/bit) & 3.2 & 7.1 \\
\hline Write speed (ns/bit) & $\begin{array}{c}\text { Soft Transition: 9.3 } \\
\text { Hard Transition: 19.2 }\end{array}$ & 7.1 \\
\hline
\end{tabular}

estimate the power and energy of DRAM based on Micron's DRAM power model [27].

To evaluate the performance of GPU systems with MLC STTRAM, we developed an in-house script with a performance model incorporated with MLC STT-RAM-based GPU memory and memory controllers. We obtain memory access statistics from real-machine profiling and feed the statistics into our performance model to estimate the memory access performance of our proposed design. To evaluate GPU processor power and energy, we employ the power statistics obtained by NVIDIA profiler [2]. We use McPAT [33] to estimate the power of memory controllers. We assume that our design does not significantly change the power of the rest of the processor. Therefore, we only show memory energy consumption and the overhead of the memory controllers. We calculate the dynamic energy of MLC STT-RAM using our energy parameters listed in Table 3 and our performance results.

\section{RESULTS}

In this section, we report and analyze our evaluation results. We compare among the following memory configurations:

- DRAM - traditional GDDR5X-based GPU memory.

- MLC STT-RAM - MLC STT-RAM-based GPU memory without our data remapping, BitLevel, and SparseCode mechanisms.

- BitLevel - MLC STT-RAM-based GPU memory with our data remapping and BitLevel mechanisms.

- BitLevel\&SparseCode - MLC STT-RAM-based GPU memory with all our proposed mechanisms.

\subsection{Performance and Energy}

Energy consumption. We only show the memory access energy consumption reduction and performance improvement in the following sections, because memory access is the main bottleneck for energy consumption and performance (Figure 1), also our proposed modifications to memory controller only introduces negligible overhead. We observe that naïvely replacing main memory with MLC STT-RAM can hardly improve energy efficiency and performance. Figure 12 and Figure 14 show that using MLC STT-RAM as main memory without our proposed mechanism only reduces memory dynamic energy around by $7 \%$.

Although MLC STT-RAM can lead to lower read energy than DRAM-based memory, it significantly increases write energy because substantial amount of writes need to be performed in two steps. For VGG-19, with our data remapping scheme and BitLevel mechanism, we reduce $26 \%$ write energy compared with the naïve replacement. Combining our SparseCode mechanism with BitLevel can reduce memory dynamic energy consumption by $45 \%$ compared to the baseline DRAM-based design, with further energy savings in both reads and writes. For AlexNet, our design can reduce memory dynamic energy consumption by $40 \%$ compared to the baseline (Figure 14). We also perform a sensitivity study on the size of data blocks in BitLevel mechanism, ranging from a single 32-bit floating point value to 128-bit floating point values. As shown in Figure 12 and Figure 14, the increase of the data block size will further reduce the memory energy consumption.

Memory access performance. We evaluate the memory access performance of our design by taking into account the latency of both memory access. Again, Figure 12 shows that naïve memory replacement with MLC STT-RAM only introduce $11 \%$ speedup on average write latency of VGG-19 memory access. Yet, the direct replacement can improve read performance by $1.4 \times$, despite the $47 \%$ write performance degradation. Our data remapping with BitLevel mechanism improves both read and write performance, leading to $30 \%$ improvement of overall memory access speedup. Combining SparseCode can further improve access performance. Overall, our design results in $76 \%$ and $70 \%$ speedup of memory access for VGG19 and AlexNet, respectively.

In-memory data characteristics. Figure 16 shows the bit-level neighbor data similarity for VGG-19. Overall, the average similarity across all layers can reach up to $67.3 \%$. As shown in Figure 5, between two maxpooling layers, convolutional layers and ReLU layers have almost same memory capacity utilization. Therefore, the number of write operations in order to store the feature maps can also be similar. Figure 17 illustrates the compression ratio of each ReLU layer with the SparseCode mechanism for VGG-19. Across all layers, the mechanism can up to $26.9 \%$ compression ratio with 128-bit value (data block) size. This leads to substantial reduction in memory accesses and capacity consumption. AlexNet also has the same trend in bit-level similarity and ReLU layer compression ratio, so we do not show them in this section.

\subsection{Memory Capacity Utilization}

In Figure 17, we present the compression ratio with three different bit-width: 32-bit, 64-bit and 128-bit, which can accommodate various requirements for precisions of different networks. When the bit-width is increasing, the compression efficiency will also be improved, because SparseCode uses a 4-bit index to represent data with longer bit length. With our proposed SparseCode mechanism, the total system's feature map compression ratio of VGG-19 and AlexNet can reach to $26.9 \%$ and $26 \%$ with 128 -bit length, respectively. 


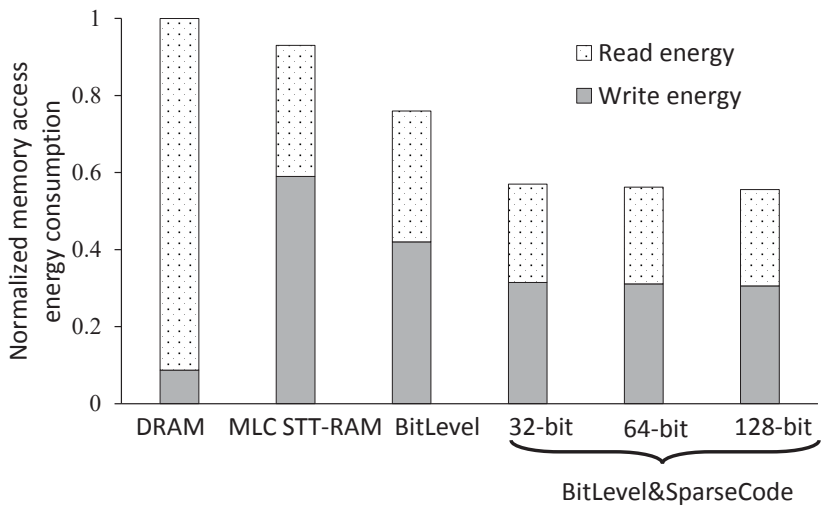

Figure 12: Energy consumption saving comparing with DRAM for VGG-19.

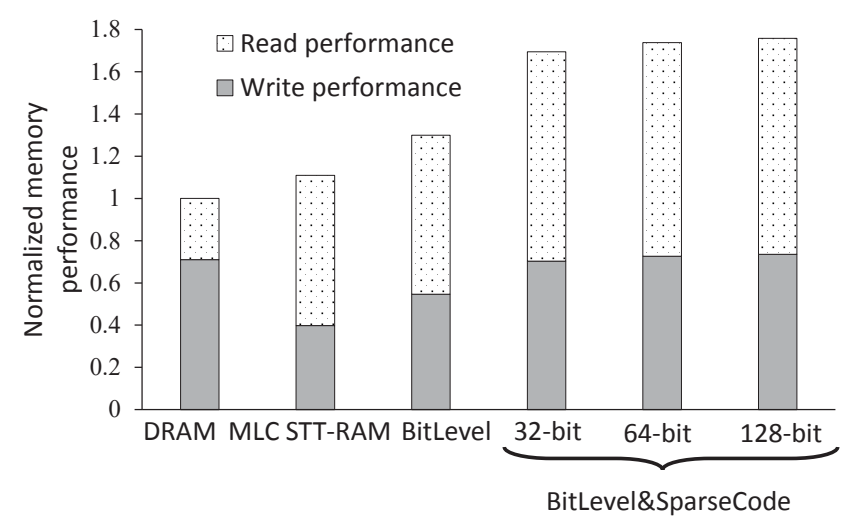

Figure 13: Performance improvement comparing with DRAM for VGG-19.

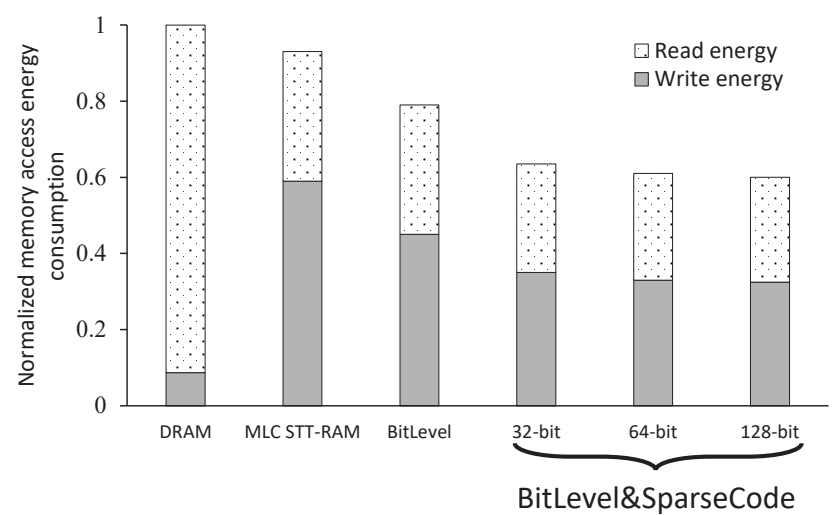

Figure 14: Energy consumption saving comparing with DRAM for AlexNet.

What is more, due to the unique cell architecture of MLC STT-RAM, with same quantity of memory cells, the memory capacity of MLC STT-RAM main memory will be doubled than traditional DRAM main memory.

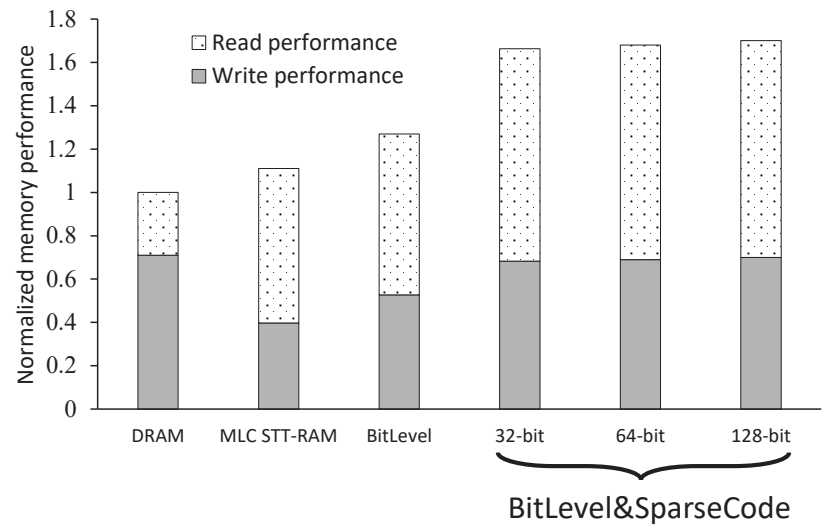

Figure 15: Performance improvement comparing with DRAM for AlexNet.

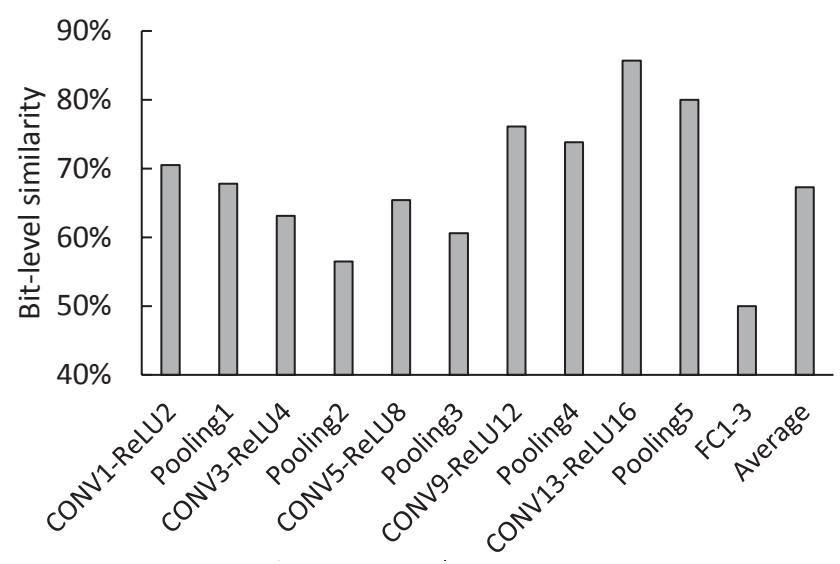

Figure 16: Bit-level similarity of every layer for VGG-19.

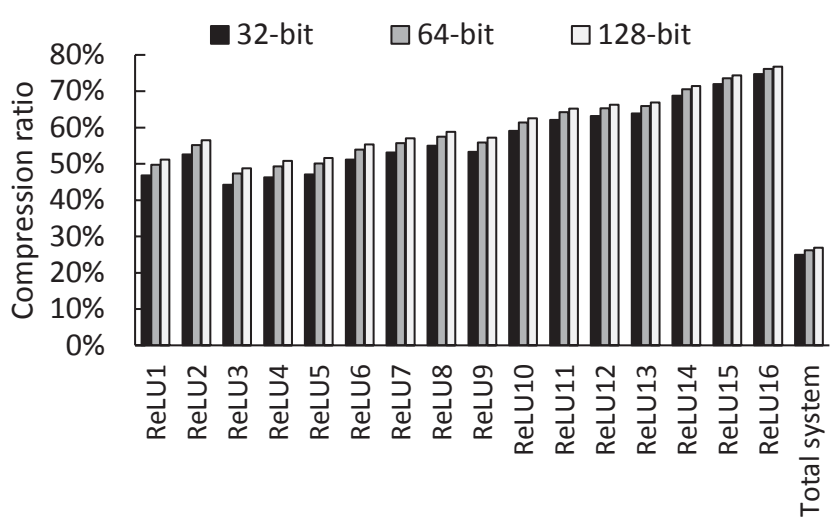

Figure 17: Compression ratio of each ReLU layer for VGG19.

\subsection{Memory Bandwidth}

Figure 1(b) shows that memory read bandwidth is higher than memory write bandwidth, so in this section we will analyze read and write bandwidth respectively. With our proposed scheme, for 
VGG-19, memory read bandwidth can be reduced to 33\% of DRAM based main memory bandwidth. However, due to the poor write performance of MLC STT-RAM, memory write bandwidth increases by $14 \%$ comparing with DRAM based main memory bandwidth.

Considering CNNs are memory read intensive applications (Figure 1(b)), total memory bandwidth of VGG-19 and AlexNet will be still lower than DRAM based main memory bandwidth with $45 \%$, $40 \%$, respectively.

\subsection{Memory Area Efficiency}

We use NVSim [18] to obtain MLC STT-RAM area model and area efficiency. With the same size of 2GB, DRAM costs area of $419 \mathrm{~mm}^{2}$ and MLC STT-RAM only costs $372 \mathrm{~mm}^{2}$. Also, the area efficiency of MLC STT-RAM is $37.8 \%$, is higher than DRAM's $25.2 \%$.

\section{RELATED WORK}

A large body of previous works endeavor to reduce memory intensity and energy consumption of neural networks. Network pruning [22-24] aims to reduce the memory consumption through pruning small valued weight connections in neural networks. Other redundancy alleviating methods exploit reduced precision [29] to reduce the number of bits required by corresponding neural networks. In addition, recent works proposed a variety of CNN inference accelerators $[12,14,15,21,34,35,37]$. These designs improve the energy efficiency and performance of neural networks. However, none of these studies focuses on the memory issues of $\mathrm{CNN}$ training. Moreover, most of previous works focus on improving the efficiency of storing the weights in neural networks. However, in training phase, most of the memory space is used by storing feature maps. Most previous works do not explore the impact of feature maps on performance and energy of CNNs. Finally, prior design with reduced precision policies can result in the decrease of CNN accuracy. Our design does not sacrifice precision, when improving memory access performance and energy efficiency.

Rhu et al. demonstrated that the increase of memory demand of neural network training phase can outpace the development of commodity GPU systems. Their study proposed a runtime memory manager to virtualize the memory usage of DNNs and schedule CPU memory and GPU memory simultaneously [36]. This study reduces the average GPU memory usage, by trading off performance, energy consumption and memory bandwidth. However, our design simultaneously improves memory energy, performance, and capacity utilization.

\section{CONCLUSION}

GPU memory capacity, bandwidth, and energy consumption are becoming critical system bottlenecks with increasingly larger-scale CNN trainings. In this paper, we propose an energy-efficient GPU memory management scheme that leverages the access asymmetry of soft and hard bits in MLC STT-RAM technology and the characteristics of image classification training workloads. The evaluation results shows that our proposed scheme can improve the performance of VGG-19 and AlexNet training by $76 \%$ and $70 \%$, respectively. Meanwhile, 45\% and 40\% GPU memory access energy and $26.9 \%$ and $26 \%$ capacity utilization of VGG-19 and AlexNet have been reduced, respectively.

\section{ACKNOWLEDGMENTS}

This work was supported in part by NSF 1652328 and 1718158, and SRC/DARPA Center for Research on Intelligent Storage and Processing-in-memory.

\section{REFERENCES}

[1] 2016. NVIDIA, cuDNN: GPU accelerated deep learning. (2016).

[2] http://docs.nvidia.com/cuda/profiler-users-guide/. NVIDIA, Profiler user's guide. (http://docs.nvidia.com/cuda/profiler-users-guide/).

[3] https://github.com/amd/OpenCL-caffe. AMD: Caffe for OpenCL. (https://github.com/amd/OpenCL-caffe).

[4] https://www.micron.com//media/documents/ products/data-sheet/dram/gddr5/ $8 \mathrm{gb}$ gddr5x sgram brief.pdf. Micron, GDDR5X SGRAM specification. (https://www.micron.com//media/documents/ products/data-sheet/dram/gddr5/ 8gb_gddr5x_sgram_brief.pdf).

[5] https://www.nvidia.com/en-us/geforce/products/. NVIDIA, GeForce GTX 1080 Ti. (https://www.nvidia.com/en-us/geforce/products/)

[6] https://www.nvidia.com/en-us/geforce/products/10series/titan-xp/. NVIDIA, TITAN Xp. (https://www.nvidia.com/en-us/geforce/products/10series/titan-xp/).

[7] http://www.image-net.org/challenges/LSVRC/. ImageNet Large Scale Visual Recognition Challenge(ILSVRC). (http://www.imagenet.org/challenges/LSVRC/).

[8] Martin Abadi, Paul Barham, and Jianmin Chen et al. 2016. TensorFlow: A system for large-scale machine learning. In 12th USENIX Symposium on Operating Systems Design and Implementation (OSDI 16). 265-283.

[9] M Aoki, H Noshiro, K Tsunoda, Y Iba, A Hatada, M Nakabayashi, A Takahashi, C Yoshida, Y Yamazaki, T Takenaga, and others. 2013. Novel highly scalable multilevel cell for STT-MRAM with stacked perpendicular MTJs. In VLSI Technology (VLSIT), 2013 Symposium on. T134-T135.

[10] Chris Burges, Tal Shaked, and Erin et al. Renshaw. 2005. Learning to rank using gradient descent. In Proceedings of the 22nd international conference on Machine learning. ACM, 89-96.

[11] Xunchao Chen, Navid Khoshavi, Ronald F DeMara, Jun Wang, Dan Huang, Wujie Wen, and Yiran Chen. 2017. Energy-Aware Adaptive Restore Schemes for MLC STT-RAM Cache. IEEE Trans. Comput. 66, 5 (2017), 786-798.

[12] Yunji Chen, Tao Luo, Shaoli Liu, Shijin Zhang, Liqiang He, Jia Wang, Ling Li, Tianshi Chen, Zhiwei Xu, Ninghui Sun, and others. 2014. Dadiannao: A machinelearning supercomputer. In Proceedings of the 47th Annual IEEE/ACM International Symposium on Microarchitecture. 609-622.

[13] Yiran Chen, Xiaobin Wang, and Wenzhong et al. Zhu. 2010. Access scheme of multi-level cell spin-transfer torque random access memory and its optimization. In Circuits and Systems (MWSCAS), 2010 53rd IEEE International Midwest Symposium on. 1109-1112.

[14] Yu-Hsin Chen, Joel Emer, and Vivienne Sze. 2016. Eyeriss: A spatial architecture for energy-efficient dataflow for convolutional neural networks. In Computer Architecture (ISCA), 2016 ACM/IEEE 43rd Annual International Symposium on. IEEE, 367-379.

[15] Ping Chi, Shuangchen Li, Cong Xu, Tao Zhang, Jishen Zhao, Yongpan Liu, Yu Wang, and Yuan Xie. 2016. Prime: A novel processing-in-memory architecture for neural network computation in reram-based main memory. In Proceedings of the 43rd International Symposium on Computer Architecture. 27-39.

[16] Ping Chi, Cong Xu, and Tao et al. Zhang. 2014. Using multi-level cell STT-RAM for fast and energy-efficient local checkpointing. In Computer-Aided Design (ICCAD), 2014 IEEE/ACM International Conference on. IEEE, 301-308.

[17] Gregory Diamos, Shubho Sengupta, Bryan Catanzaro, Mike Chrzanowski, Adam Coates, Erich Elsen, Jesse Engel, Awni Hannun, and Sanjeev Satheesh. 2016. Persistent RNNs: Stashing Recurrent Weights On-chip. In Proceedings of the 33rd International Conference on International Conference on Machine Learning - Volume 48.

[18] Xiangyu Dong, Cong Xu, Norm Jouppi, and Yuan Xie. 2014. NVSim: A circuitlevel performance, energy, and area model for emerging non-volatile memory. In Emerging Memory Technologies. Springer, 15-50.

[19] Xiaohua Lou et al. 2008. Demonstration of multilevel cell spin transfer switching in $\mathrm{MgO}$ magnetic tunnel junctions. Applied Physics Letters (2008).

[20] Yaojun Zhang et al. 2012. Multi-level cell STT-RAM: Is it realistic or just a dream?. In ICCAD.

[21] Song Han, Xingyu Liu, and Huizi Mao et al. 2016. EIE: efficient inference engine on compressed deep neural network. In ISCA. 243-254.

[22] Song Han, Huizi Mao, and William J Dally. 2016. Deep compression: Compressing deep neural networks with pruning, trained quantization and huffman coding. International Conference on Learning Representations (ICLR) (2016).

[23] Stephen José Hanson and Lorien Y Pratt. 1989. Comparing biases for minimal network construction with back-propagation. In Advances in neural information processing systems. 177-185. 
[24] Babak Hassibi and David G Stork. 1993. Second order derivatives for network pruning: Optimal brain surgeon. In Advances in neural information processing systems. $164-171$.

[25] Kaiming He, Xiangyu Zhang, Shaoqing Ren, and Jian Sun. 2016. Deep residual learning for image recognition. In Proceedings of the IEEE conference on computer vision and pattern recognition. 770-778.

[26] T. Ishigaki et al. 2010. A multi-level-cell spin-transfer torque memory with series-stacked magnetotunnel junctions. In VLSIT.

[27] Jeff Janzen. The Micron system-power calculator. (????) http://www.micron.com/products/dram/syscalc.html

[28] Yangqing Jia, Evan Shelhamer, and Jeff Donahue et al. 2014. Caffe: Convolutional Architecture for Fast Feature Embedding. arXiv preprint arXiv:1408.5093 (2014).

[29] Patrick Judd, Jorge Albericio, Tayler Hetherington, Tor Aamodt, Natalie Enright Jerger, Raquel Urtasun, and Andreas Moshovos. 2015. Reduced-precision strategies for bounded memory in deep neural nets. arXiv preprint arXiv:1511.05236 (2015).

[30] Heehoon Kim, Hyoungwook Nam, Wookeun Jung, and Jaejin Lee. 2017. Performance Analysis of CNN Frameworks for GPUs. In ISPASS.

[31] A. Krizhevsky. 2014. One Weird Trick For Parallelizing Convolutional Neural Networks. In arxiv.org.

[32] Alex Krizhevsky, Ilya Sutskever, and Geoffrey E Hinton. 2012. Imagenet classification with deep convolutional neural networks. In Advances in neural information processing systems. 1097-1105.

[33] Sheng Li, Jung Ho Ahn, Richard D. Strong, Jay B. Brockman, Dean M. Tullsen and Norman P. Jouppi. 2009. McPAT: An Integrated Power, Area, and Timing Modeling Framework for Multicore and Manycore Architectures. In Proceedings of the 42Nd Annual IEEE/ACM International Symposium on Microarchitecture. 469-480.

[34] Angshuman Parashar, Minsoo Rhu, and Anurag et al. Mukkara. 2017. SCNN: An Accelerator for Compressed-sparse Convolutional Neural Networks. In ISCA

[35] Brandon Reagen, Paul Whatmough, Robert Adolf, Saketh Rama, Hyunkwang Lee Sae Kyu Lee, José Miguel Hernández-Lobato, Gu-Yeon Wei, and David Brooks. 2016. Minerva: Enabling low-power, highly-accurate deep neural network accelerators. In Proceedings of the 43rd International Symposium on Computer Architecture. 267-278.

[36] Minsoo Rhu, Natalia Gimelshein, and Jason Clemons et al. 2016. vDNN: Virtualized Deep Neural Networks for Scalable, Memory-Efficient Neural Network Design. In MICRO.

[37] Ali Shafiee, Anirban Nag, Naveen Muralimanohar, Rajeev Balasubramonian, John Paul Strachan, Miao Hu, R Stanley Williams, and Vivek Srikumar. 2016. ISAAC: A convolutional neural network accelerator with in-situ analog arithmetic in crossbars. In Proceedings of the 43rd International Symposium on Computer Architecture. 14-26.

[38] Karen Simonyan and Andrew Zisserman. 2014. Very Deep Convolutional Networks for Large-Scale Image Recognition. arXiv preprint arXiv:1409.1556 (2014).

[39] Christian Szegedy, Wei Liu, and Yangqing Jia et al. 2015. Going deeper with convolutions. In Proceedings of the IEEE Conference on Computer Vision and Pattern Recognition. 1-9.

[40] The Next Platform. 2016. Baidu Eyes Deep Learning Strategy in Wake of New GPU Options. In www.nextplatform.com.

[41] Theano Development Team. 2016. Theano: A Python framework for fast computation of mathematical expressions. arXiv e-prints abs/1605.02688 (May 2016). 